\title{
Entropy Generation and Integral Inequalities
}

\author{
Xiaowei $\operatorname{Tian}^{1,2}$ and Liqiu Wang ${ }^{1,2 *}$
}

As a typical irreversible process, the heat conduction in rectangles results in entropy generation. As time tends to infinity, this entropy generation evolves into a finite value when the heat conduction comes from the initial temperature distribution, but into infinity whenever a positively-averaged heat source is involved. An application of the second law of thermodynamics to this process leads to nine integral inequalities which are important for studying heat-conduction equations and for uncovering some basic features of the total multiplicity and the Boltzmann entropy. The work correlates the second law of thermodynamics in thermodynamics and integral inequalities in mathematics, and inspires the future work in offering some fundamental insights into our future.

Keywords: Entropy generation; Integral inequalities; Second law of thermodynamics; Heat conduction

Received 15 March 2019, Accepted 19 May 2019

DOI: $10.30919 /$ esee8c295

\section{Introduction}

Energy is defined as the ability or the potential of system to cause changes. ${ }^{1,2}$ It can exist in numerous forms such as thermal, mechanical, kinetic, potential, electric, magnetic, chemical, and nuclear; their sum constitutes the total energy of a system., ${ }^{1,2}$ During every practical process, energy is conserved following the first law of thermodynamics, but degraded according to the second law of thermodynamics. ${ }^{1,2}$ The conservation in energy quantity is represented by the heat-conduction equation for heat-conduction processes in which the classical Fourier's law of heat conduction is normally used as the constitutive relation of heat flux density. ${ }^{3-5}$ The degradation comes from the entropy generation occurring in any practical process like heat conduction because of irreversibilities. $^{1,2,6}$

The solution of the heat-conduction equation with its initial and boundary conditions yields the temperature field, the temperature at any location and any time instant in heat conduction., ${ }^{3,4}$ The Fourier's law of heat conduction is then applied to find heat-transfer rate and the way to enhance/reduce it. ${ }^{3,4}$ With the known temperature field, the entropy generation in heat conduction becomes available as well by using the thermodynamic relations from the first and second laws of thermodynamics. ${ }^{1,2,6}$ With the available entropy generation, we can then develop integral inequalities and examine features of heat-conduction equations by applying the second law of thermodynamics in the form that the entropy generation $S_{\text {gen }}$ of a system during heat conduction always increases, or, in the limiting case of a reversible process, remains constant, i.e., $d S_{\text {gen }} / d t \geq 0$ with $t$ being the time and $S_{g e n}\left(t_{2}\right)$ $\geq S_{\text {gen }}\left(t_{1}\right)$ for all $t_{2} \geq t_{1}{ }^{1,2,6}$ The entropy at state 1 can be defined as, $S_{1}=$

\footnotetext{
'Department of Mechanical Engineering, the University of Hong Kong, Hong Kong

${ }^{2} H K U$-Zhejiang Institute of Research and Innovation (HKU-ZIRI), Hangzhou, China

*E-mail: lqwang@hku.hk
}

$C Q_{0 \mathrm{R}}^{10}$, where $Q_{\mathrm{OR}}^{10}$ is the heat exchanged between the system and the environment undergoing a totally reversible process from State 1 to the reference state 0 , and $C$ can be any positive constants. ${ }^{7} Q_{0 R}^{10}$ is processindependent and represents the part of system energy that cannot be used to do useful work. ${ }^{7}$ Constant $C$ was introduced for recovering the classical definition of entropy. However, it is more convenient and desirable for illustrating the physical meaning of entropy and performing entropy analysis with $C$ being 1 (no unit) such that entropy being with an energy unit. This definition is identical to the classical one by choosing the constant $C$ as the inverse of the environment temperature (so that the classical definition of entropy with a unit of $\mathrm{J} / \mathrm{K})^{7}$. Any entropy generation will thus downgrade the energy quality. It is therefore important to study the entropy generation in general, and its boundedness in particular.

We examine $d S_{g e n} / d t$ and $\lim _{t \rightarrow \infty} S_{g e n}$ and derive mathematical inequalities with heat conduction in rectangles. This kind of analysis is recently initiated with heat conduction in cylinders, ${ }^{8}$ is limited in the literature $^{8}$ and differs from conventional second-law analysis that has been made mainly for performance evaluation, weak-component identification or performance optimization. ${ }^{9-30}$

\section{Temperature field, entropy generation and integral inequalities}

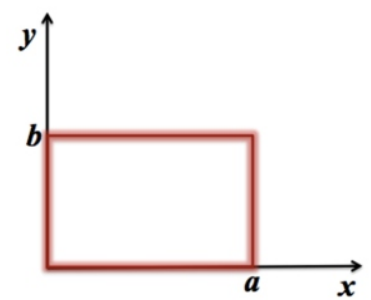

Fig. 1 Heat conduction in adiabatic rectangles and Cartesian coordinate system. 
Consider heat conduction in a rectangle D of width $a$ and height $b$ subject to specified temperature gradient atthe rectangle boundary. Material properties are assumed to be constant. Note that the effect of nonhomogeneous boundary condition is representable by source and initial terms. ${ }^{3,6}$ We thus limit our attention to the following initialboundary value problem under homogeneous boundary conditions in Cartesian coordinates, shown in Fig. 1, without loss of the generality:, 4, 31

$$
\begin{aligned}
& \left\{\begin{array}{l}
T_{\mathrm{t}}=a_{0}^{2}\left(T_{x x}+T_{y y}\right)+f(x, y, t), \mathrm{D} \times(0,+\infty) \\
\left.T_{x}\right|_{x=0, a}=\left.T_{y}\right|_{y=0, b}=0 \\
\left.T\right|_{t=0}=\varphi(x, y)
\end{array}\right. \\
& \text { D: } 0<x<a, 0<y<b
\end{aligned}
$$

where $t$ and $T$ are time and temperature, respectively. $a_{0}^{2}$ is the thermal diffusivity. $\varphi(x, y)$ is the initial temperature distribution over the rectangle. $f(x, y, t)$ is the rate of volumetric heat generation inside the rectangle per unit specific capacity of the material. The heat generation may be due to chemical, electrical,gammy-ray, nuclear, or other sources that may be a function of time and/or position. Fig. 2 examples the heat conduction driven by the initial temperature distribution, the internal source and the both, respectively, the readers are refer to ${ }^{31}$ for more practical examples.

\subsection{Heat conduction initiated by the initial temperature distribution}

For the heat conduction driven by the initial temperature distribution, $f$ ( $x, y, t)=0$, and Eq. (1) reduces into

$$
\left\{\begin{array}{l}
T_{\mathrm{t}}=a_{0}^{2}\left(T_{x x}+T_{y y}\right), \mathrm{D} \times(0,+\infty) \\
\left.T_{x}\right|_{x=0, a}=\left.T_{y}\right|_{y=0, b}=0 \\
\left.T\right|_{t=0}=\varphi(x, y)
\end{array}\right.
$$

Applying generalized Fourier extension to the solution of (2) yields, ${ }^{3}$

$$
T(x, y, t)=\sum_{m, n=0}^{\infty} T(t) a_{m n} \cos \frac{m \pi x}{a} \cos \frac{n \pi y}{b}
$$

Because

$$
T_{t}=\sum_{m, n=0}^{\infty} T_{t}(t) a_{m n} \cos \frac{m \pi x}{a} \cos \frac{n \pi y}{b}
$$

$$
\begin{aligned}
& T_{x x}=\sum_{m, n=0}^{\infty}-\left(\frac{m \pi}{a}\right)^{2} T(t) a_{m n} \cos \frac{m \pi x}{a} \cos \frac{n \pi y}{b} \\
& T_{y y}=\sum_{m, n=0}^{\infty}-\left(\frac{n \pi}{b}\right)^{2} T(t) a_{m n} \cos \frac{m \pi x}{a} \cos \frac{n \pi y}{b}
\end{aligned}
$$

Eq. (2) becomes

$$
\begin{gathered}
\sum_{m, n=0}^{\infty} T_{t}(t) a_{m n} \cos \frac{m \pi x}{a} \cos \frac{n \pi y}{b} \\
+\sum_{m, n=0}^{\infty}\left(\frac{m \pi a_{0}}{a}\right)^{2} T(t) a_{m n} \cos \frac{m \pi x}{a} \cos \frac{n \pi y}{b} \\
+\sum_{m, n=0}^{\infty}\left(\frac{n \pi a_{0}}{b}\right)^{2} T(t) a_{m n} \cos \frac{m \pi x}{a} \cos \frac{n \pi y}{b}=0
\end{gathered}
$$

Let $\omega_{m n}^{2}=\left(\frac{m \pi a_{0}}{a}\right)^{2}+\left(\frac{n \pi a_{0}}{b}\right)^{2}$, thus

$$
\sum_{m, n=0}^{\infty} a_{m n}\left[T_{t}(t)+\omega_{m n}^{2} T(t)\right] \cos \frac{m \pi x}{a} \cos \frac{n \pi y}{b}=0
$$

Therefore, one has, because $a_{m n} \neq 0$,

$$
T_{t}(t)+\omega_{m n}^{2} T(t)=0
$$

The solution of Eq. (9) reads, ${ }^{3}$

$$
T_{m n}(t)=c_{m n} e^{-\omega_{m n}^{2} t}, c_{m n} \text { is constant }
$$

Define constant, $A_{m n}=a_{m n} c_{m n}$ then substituting Eq. (10) into Eq. (3) yields the solution of Eq. (2)

$$
T(x, y, t)=\sum_{m, n=0}^{\infty} A_{m n} e^{-\omega_{m n}^{2} t} \cos \frac{m \pi x}{a} \cos \frac{n \pi y}{b}
$$

By using the initial condition and the orthogonality of function group $\left\{\cos \frac{m \pi x}{a}\right\}, x \in(0, a)$ and $\left\{\cos \frac{n \pi y}{b}\right\}, y \in(0, b)$, one arrives at
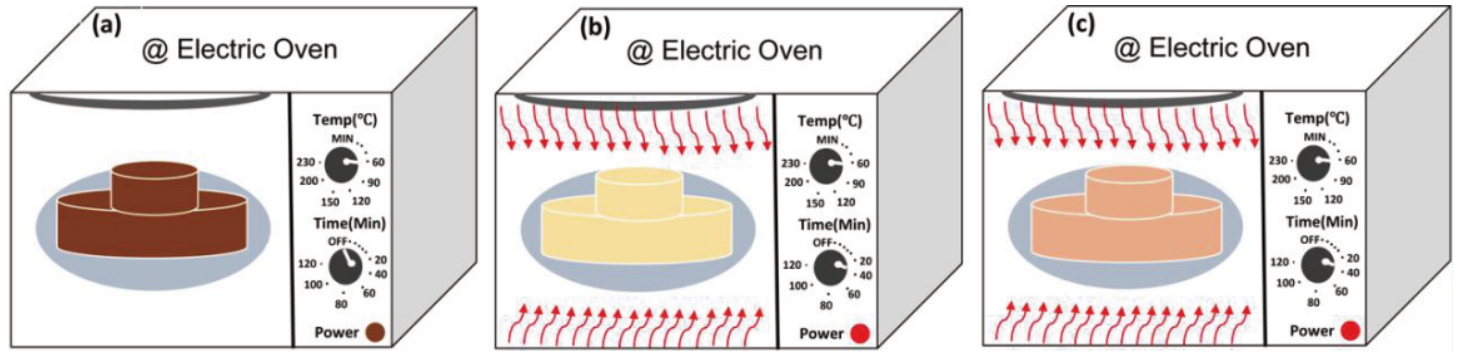

Fig. 2 Heat conduction in oven: (a) heat conduction driven by the initial temperature distribution after switching off the power, (b) heat conduction driven by the internal source when the power is first switched on, (c) heat conduction driven by the initial temperature distribution and the internal source after re-switching on the power. 


$$
\begin{gathered}
\varphi(x, y)=\sum_{m, n=0}^{\infty} A_{m n} \cos \frac{m \pi x}{a} \cos \frac{n \pi y}{b} \\
A_{m n}=\frac{1}{M_{m n}} \int_{0}^{b} \int_{0}^{a} \varphi(x, y) \cos \frac{m \pi x}{a} \cos \frac{n \pi y}{b} d x d y
\end{gathered}
$$

where

$$
M_{m n}=\left\{\begin{array}{l}
a b, m=n=0 \\
\frac{a b}{2}, m=0, n=1 \text { or } m=0, n=1 \\
\frac{a b}{4}, m, n=1,2,3, \ldots
\end{array}\right.
$$

Therefore, the temperature distribution $T_{\varphi}(x, y, t)$ due to initial temperature distribution $\varphi(x, y)$ can be expressed as

$$
\left\{\begin{array}{l}
T_{\varphi}(x, y, t)=\sum_{m, n=0}^{\infty} A_{m n} e^{-\omega_{m n}^{2} t} \cos \frac{m \pi x}{a} \cos \frac{n \pi y}{b} \\
A_{m n}=\frac{1}{M_{m n}} \iint_{D} \varphi(x, y) \cos \frac{m \pi x}{a} \cos \frac{n \pi y}{b} d A, m, n=0,1,2, \ldots
\end{array}\right.
$$

where $A=a b$ is the area of domain $\mathrm{D}$.

The total entropy in the rectangle is, by using the first ds equation (the one of two fundamental equations for calculating the entropy change in engineering thermodynamics) from the first and second law of thermodynamics, ${ }^{1,2,6}$

$$
\begin{aligned}
& S_{\varphi}(t)=\rho C_{V} \int_{0}^{b} \int_{0}^{a} \ln \left[T_{\varphi}(x, y, t)\right] d x d y \\
& \quad=\rho C_{V} \iint_{D} \ln \left(\sum_{m, n=0}^{\infty} A_{m n} e^{-\omega_{m n}^{2} t} \cos \frac{m \pi x}{a} \cos \frac{n \pi y}{b}\right) d \mathrm{~A}
\end{aligned}
$$

Note that $S_{\varphi}(t)$ is also the total entropy generation for the case of adiabatic boundary conditions with vanished thermal entropy flux. ${ }^{1,6}$

By applying Eq. (16) and noting that $\omega_{m n}^{2}>0$ for $m+n \neq 0$, we obtain

$$
\begin{aligned}
& \lim _{t \rightarrow+\infty} S_{\varphi}(t) \\
= & \rho C_{V} \int_{0}^{b} \int_{0}^{a} \lim _{t \rightarrow+\infty} \ln \left(\sum_{m, n=0}^{\infty} A_{m n} e^{-\omega_{m n}^{2} t} \cos \frac{m \pi x}{a} \cos \frac{n \pi y}{b}\right) d x d y \\
= & \rho C_{V} \int_{0}^{b} \int_{0}^{a} \lim _{t \rightarrow+\infty} \ln \left(A_{00}+\sum_{m, n=0, m+n \neq 0}^{\infty}\right. \\
& \left.A_{m n} e^{-\omega_{m n}^{2} t} \cos \frac{m \pi x}{a} \cos \frac{n \pi y}{b}\right) d x d y \\
= & \rho C_{V} \int_{0}^{b} \int_{0}^{a} \ln A_{00} d x d y=\rho C_{V} \iint_{D} \ln A_{00} d A
\end{aligned}
$$

By using Eqs. (13) and (14),

$$
A_{00}=\frac{1}{A} \iint_{\mathrm{D}} \varphi(x, y) d \mathrm{~A}
$$

which is the average of the initial temperature distribution $\varphi(x, y)$ over the rectangle. Therefore,

$$
\begin{aligned}
& \lim _{t \rightarrow+\infty} S_{\varphi}(t) \\
& =\rho C_{V} \iint_{D} \ln \left[\frac{1}{A} \iint_{D} \varphi(x, y) d \mathrm{~A}\right] d A \\
& =\rho C_{V} A \ln \left[\frac{1}{A} \iint_{D} \varphi(x, y) d \mathrm{~A}\right]
\end{aligned}
$$

The limit of $S_{q}(t)$ is thus bounded as time trends to infinity and equals to system total entropy with the temperature being the average of the initial temperature distribution over the whole rectangle. Note that,

$$
\begin{aligned}
& \frac{d S_{\varphi}(t)}{d t}=\rho C_{v} \int_{0}^{b} \int_{0}^{a} \frac{1}{T_{\varphi}(x, y, t)} \frac{\partial T_{\varphi}(x, y, t)}{\partial t} d x d y \\
& \frac{\partial T_{\varphi}(x, y, t)}{\partial t}=\sum_{m, n=0}^{\infty} A_{m n}\left(-\omega_{m n}^{2}\right) e^{-\omega_{m n}^{2} t} \cos \frac{m \pi x}{a} \cos \frac{n \pi y}{b}
\end{aligned}
$$

Applying the principle of entropy increase $d S_{\varphi}(t) / d t \geq 0$ leads to,

$$
\iint_{\mathrm{D}} \frac{\sum_{m, n=0}^{\infty} A_{m n}\left(-\omega_{m n}^{2}\right) e^{-\omega_{m n}^{2} t} \cos \frac{m \pi x}{a} \cos \frac{n \pi y}{b}}{\sum_{m, n=0}^{\infty} A_{m n} e^{-\omega_{m n}^{2} t} \cos \frac{m \pi x}{a} \cos \frac{n \pi y}{b}} d \mathrm{~A} \geq 0
$$

As $d S_{\varphi}(t) / d t \geq 0$, one also has

$$
S_{\varphi}\left(t_{1}\right) \leq S_{\varphi}\left(t_{2}\right) \quad \forall 0<t_{1} \leq t_{2}<+\infty
$$

or

$$
\int_{0}^{b} \int_{0}^{a} \ln \left[T_{\varphi}\left(x, y, t_{1}\right)\right] d x d y \leq \int_{0}^{b} \int_{0}^{a} \ln \left[T_{\varphi}\left(x, y, t_{2}\right)\right] d x d y
$$

This yields the integral inequality

$$
\begin{aligned}
& \iint_{\mathrm{D}} \ln \left(\sum_{m, n=0}^{\infty} A_{m n} e^{-\omega_{m n}^{2} t_{1}} \cos \frac{m \pi x}{a} \cos \frac{n \pi y}{b}\right) d \mathrm{~A} \\
& \quad \leq \iint_{\mathrm{D}} \ln \left(\sum_{m, n=0}^{\infty} A_{m n} e^{-\omega_{m n}^{2} t_{2}} \cos \frac{m \pi x}{a} \cos \frac{n \pi y}{b}\right) d \mathrm{~A}
\end{aligned}
$$


Note that

$S_{\varphi}(0)=\rho C_{V} \int_{0}^{b} \int_{0}^{a} \ln \left[T_{\varphi}(x, y, t)\right] d x d y=\rho C_{V} \iint_{D} \ln \varphi(x, y) d \mathrm{~A}$

and

$$
S_{\varphi}(+\infty)=\rho C_{V} A \ln \left[\frac{1}{A} \iint_{\mathrm{D}} \varphi(x, y) d \mathrm{~A}\right]
$$

Applying $S_{\varphi}(0) \leq S_{\varphi}(+\infty)$ leads to

$$
\rho C_{V} \iint_{\mathrm{D}} \ln \varphi(x, y) d \mathrm{~A} \leq \rho C_{V} A \ln \left[\frac{1}{A} \iint_{\mathrm{D}} \varphi(x, y) d \mathrm{~A}\right]
$$

that is

$$
\frac{1}{A} \iint_{\mathrm{D}} \ln \varphi(x, y) d \mathrm{~A} \leq \ln \left[\frac{1}{A} \iint_{\mathrm{D}} \varphi(x, y) d \mathrm{~A}\right]
$$

which is the two-dimensional extension over the rectangle of wellknown Arithmetic-mean---geometric-mean inequality ${ }^{32,33}$ and can be proven mathematically as following as well.

By the third law of thermodynamics, $\varphi(x, y)=T_{\varphi}(x, y, 0)>0$ with $T_{\varphi}$ being the temperature during the heat conduction driven exclusively by the initial temperature distribution $\varphi(x, y)$. Divide $D$ into $N$ area elements $\Delta \sigma_{i}, i=1,2, \ldots, N$, of equal area. For $\forall\left(\xi_{i}, \eta_{i}\right) \in \Delta \sigma_{i}$, one has $N$ positive numbers:

$$
\varphi\left(\xi_{i}, \eta_{i}\right), i=1,2, \ldots, N
$$

Therefore,

$$
\sqrt[N]{\prod_{i=1}^{N} \varphi\left(\xi_{i}, \eta_{i}\right) \leq \frac{\sum_{i=1}^{N} \varphi\left(\xi_{i}, \eta_{i}\right)}{N}}
$$

and

$$
\ln \sqrt[N]{\prod_{i=1}^{N} \varphi\left(\xi_{i}, \eta_{i}\right)} \leq \ln \frac{\sum_{i=1}^{N} \varphi\left(\xi_{i}, \eta_{i}\right)}{N}
$$

or

$$
\frac{1}{N} \sum_{i=1}^{N} \ln \varphi\left(\xi_{i}, \eta_{i}\right) \leq \ln \frac{\sum_{i=1}^{N} \varphi\left(\xi_{i}, \eta_{i}\right)}{N}
$$

which can be written as

$$
\frac{1}{A}\left[\sum_{i=1}^{N} \ln \varphi\left(\xi_{i}, \eta_{i}\right)\right] \frac{A}{N} \leq \ln \left[\frac{1}{A} \sum_{i=1}^{N} \varphi\left(\xi_{i}, \eta_{i}\right) \frac{A}{N}\right]
$$

Note that $A / N=\Delta x_{i} \Delta y_{i}$, one has

$$
\frac{1}{A}\left[\sum_{i=1}^{N} \ln \varphi\left(\xi_{i}, \eta_{i}\right) \Delta x_{i} \Delta y_{i}\right] \leq \ln \left[\frac{1}{A} \sum_{i=1}^{N} \varphi\left(\xi_{i}, \eta_{i}\right) \Delta x_{i} \Delta y_{i}\right]
$$

$$
\iint_{D} f(x, y) d A=\lim _{N \rightarrow \infty} \sum_{i=1}^{N} f\left(\xi_{i}, \eta_{i}\right) \Delta x_{i} \Delta y_{i}
$$

and

$\frac{1}{A} \lim _{N \rightarrow \infty}\left[\sum_{i=1}^{N} \ln \varphi\left(\xi_{i}, \eta_{i}\right) \Delta x_{i} \Delta y_{i}\right] \leq \ln \left\{\frac{1}{A} \lim _{N \rightarrow \infty}\left[\sum_{i=1}^{N} \varphi\left(\xi_{i}, \eta_{i}\right) \Delta x_{i} \Delta y_{i}\right]\right\}(36)$

Therefore, one arrives at Eq. (26),

$$
\frac{1}{A} \iint_{\mathrm{D}} \ln \varphi(x, y) d \mathrm{~A} \leq \ln \left[\frac{1}{A} \iint_{\mathrm{D}} \varphi(x, y) d \mathrm{~A}\right]
$$

While Eq. (28) is developed from the $2^{\text {nd }}$ law of thermodynamics with $\varphi(x, y)$ being the initial temperature distribution, it is actually valid for any positive function $\varphi(x, y)$. Consider the total multiplicity $\Omega$ of a macrostate, the number of microstates that compose the macrostate, in the Boltzmann entropy $S=k \ln \Omega$ with $k$ being the Boltzmann's constant. ${ }^{3436}$ Eq. (28) leads to,

$$
\begin{gathered}
\frac{1}{A} \iint_{\mathrm{D}} \ln \Omega d \mathrm{~A} \leq \ln \left[\frac{1}{A} \iint_{\mathrm{D}} \Omega d \mathrm{~A}\right] \\
\frac{1}{A} \iint_{\mathrm{D}} k \ln \Omega d \mathrm{~A} \leq k \ln \left[\frac{1}{A} \iint_{\mathrm{D}} \Omega d \mathrm{~A}\right]
\end{gathered}
$$

Therefore, the average of entropy over any domain can never be larger than the entropy calculated with the average of multiplicity over the domain.

\subsection{Heat conduction driven by the internal source}

For the heat conduction driven exclusively by the internal source, $\varphi(x, y)$ $=0$ and Eq. (1) reduces

$\left\{\begin{array}{l}T_{\mathrm{t}}=a_{0}^{2}\left(T_{x x}+T_{y y}\right)+f(x, y, t), \mathrm{D} \times(0,+\infty) \\ \left.T_{x}\right|_{x=0, a}=\left.T_{y}\right|_{y=0, b}=0 \\ \left.T\right|_{t=0}=0\end{array}\right.$

D: $0<x<a, 0<y<b$

Note that, ${ }^{3}$

$$
f(x, y, t)=\int_{0}^{t} f(x, y, \tau) \delta(\tau-t) d \tau
$$

where $\delta$ stands for the $\delta$ function. The solution of Eq. (40) is thus, by the superposition principle,

$$
T_{f}(x, y, t)=\int_{0}^{t} U(x, y, t, \tau) d \tau
$$

in which $U(x, y, t, \tau)$ is the solution of

$$
\left\{\begin{array}{l}
U_{\mathrm{t}}=a_{0}^{2}\left(U_{x x}+U_{y y}\right)+f(x, y, \tau) \delta(\tau-t) \\
\left.U_{x}\right|_{x=0, a}=\left.U_{y}\right|_{y=0, b}=0 \\
\left.U\right|_{t=0}=0
\end{array}\right.
$$


or

$$
\left\{\begin{array}{l}
U_{\mathrm{t}}=a_{0}^{2}\left(U_{x x}+U_{y y}\right) \\
\left.U_{x}\right|_{x=0, a}=\left.U_{y}\right|_{y=0, b}=0 \\
\left.U\right|_{t=\tau}=f(x, y, \tau)
\end{array}\right.
$$

By replacing $t$ and $\varphi(x, y)$ in Eq. (15) with $t-\tau$ and $f(x, y, \tau)$, respectively, the solution of Eq. (42) reads,

$$
U(x, y, t, \tau)=\sum_{m, n=0}^{\infty} B_{m n} e^{-\omega_{m n}^{2}(t-\tau)} \cos \frac{m \pi x}{a} \cos \frac{n \pi y}{b}
$$

Thus,

$$
\left\{\begin{array}{l}
T_{f}(x, y, t)=\int_{0}^{t} \sum_{m, n=0}^{\infty} B_{m n} e^{-\omega_{m n}^{2}(t-\tau)} \cos \frac{m \pi x}{a} \cos \frac{n \pi y}{b} d \tau \\
B_{m n}=\frac{1}{M_{m n}} \int_{0}^{b} \int_{0}^{a} f(\xi, \eta, \tau) \cos \frac{m \pi \xi}{a} \cos \frac{n \pi \eta}{b} d \xi d \eta, m, n=0,1,2,3, \ldots
\end{array}\right.
$$

where $T_{f}$ stands for the temperature for heat conduction driven exclusively by the internal heat source and can also be rewritten as, ${ }^{3}$

$T_{f}(x, y, t)=\int_{0}^{t} \int_{0}^{b} \int_{0}^{a} G(x, \xi, y, \eta, t-\tau) f(\xi, \eta, \tau) d \xi d \eta d \tau$

where

$$
\begin{aligned}
& G(x, \xi, y, \eta, t-\tau) \\
& =\sum_{m, n=0}^{\infty} e^{-\omega_{m n}^{2}(t-\tau)} \cos \frac{m \pi x}{a} \cos \frac{m \pi \xi}{a} \cos \frac{n \pi y}{b} \cos \frac{n \pi \eta}{b}
\end{aligned}
$$

is the Green function ${ }^{3}$.

The total entropy in the rectangle at time instant $t$ is, which is also the entropy generation up to time instant $t,^{1,2,6}$

$$
\begin{aligned}
& S_{f}(t)=\rho C_{V} \int_{0}^{b} \int_{0}^{a} \ln \left[T_{f}(x, y, t)\right] d x d y \\
= & \rho C_{V} \iint_{\mathrm{D}} \ln \left[\int_{0}^{t} \sum_{m, n=0}^{\infty} B_{m n} e^{-\omega_{m n}^{2}(t-\tau)} \cos \frac{m \pi x}{a} \cos \frac{n \pi y}{b} d \tau\right] d \mathrm{~A}
\end{aligned}
$$

By applying Eq.(48)

$$
\begin{aligned}
& \lim _{t \rightarrow+\infty} S_{f}(t)=\rho C_{v} \int_{0}^{b} \int_{0}^{a} \ln \left[\lim _{t \rightarrow+\infty} T_{f}(x, y, t)\right] d x d y \\
= & \rho C_{V} \iint_{\mathrm{D}} \ln \left\{\lim _{t \rightarrow+\infty}\left[\int_{0}^{t} \sum_{m, n=0}^{\infty} B_{m n} e^{-\omega_{m n}^{2}(t-\tau)} \cos \frac{m \pi x}{a} \cos \frac{n \pi y}{b} d \tau\right]\right\} d \mathrm{~A}
\end{aligned}
$$

which can be unbounded for some internal heat sources [see the proof in the part regarding $S_{f}(+\infty)$, Eq. (70)].

Also,

$$
\frac{d S_{f}(t)}{d t}=\rho C_{v} \int_{0}^{b} \int_{0}^{a} \frac{1}{T_{f}(x, y, t)} \frac{\partial T_{f}(x, y, t)}{\partial t} d x d y \geq 0
$$

Let $F(x, y, t, \tau)=\sum_{m, n=0}^{\infty} B_{m n} e^{-\omega_{m n}^{2}(t-\tau)} \cos \frac{m \pi x}{a} \cos \frac{n \pi y}{b}$. By applying the rule of taking derivatives of integral with respect to its parameters, one has

$$
\frac{\partial T_{f}(x, y, t)}{\partial t}=\int_{0}^{t} \frac{\partial F(x, y, t, \tau)}{\partial t} d \tau+\left.F(x, y, t, \tau)\right|_{\tau=\mathrm{t}}
$$

where

$$
\begin{aligned}
& \frac{\partial F(x, y, t, \tau)}{\partial t} \\
&= \sum_{m, n=0}^{\infty} B_{m n}\left(-\omega_{m n}^{2}\right) e^{-\omega_{m n}^{2}(t-\tau)} \cos \frac{m \pi x}{a} \cos \frac{n \pi y}{b} \\
&= \sum_{m n}\left(-\omega_{m n}^{2}\right) e^{-\omega_{m n}^{2}(t-\tau)} \\
& \sum D_{m n}=\sum_{m, n=0}^{\infty} B_{m n} \cos \frac{m \pi x}{a} \cos \frac{n \pi y}{b} \\
&\left.F(x, y, t, \tau)\right|_{\tau=\mathrm{t}}=f(x, y, t)
\end{aligned}
$$

One thus has

$$
\frac{\partial T_{f}(x, \mathrm{y}, t)}{\partial t}=\int_{0}^{t} \sum D_{m n}\left(-\omega_{m n}^{2}\right) e^{-\omega_{m n}^{2}(t-\tau)} d \tau+f(x, y, t)
$$

By the second law of thermodynamics,

$$
\frac{d S_{f}(t)}{d t} \geq 0
$$

One has thus, by noting that $\rho C_{V}>0$,

$\iint_{D} \frac{\int_{0}^{t} \sum D_{m n}\left(-\omega_{m n}^{2}\right) e^{-\omega_{m n}^{2}(t-\tau)} d \tau+f(x, y, t)}{\int_{0}^{t} \sum_{m, n=0}^{\infty} B_{m n} e^{-\omega_{m n}^{2}(t-\tau)} \cos \frac{m \pi x}{a} \cos \frac{n \pi y}{b} d \tau} d A \geq 0$

As $d S_{f}(t) / d t \geq 0$, one also has

$$
S_{f}\left(t_{1}\right) \leq S_{f}\left(t_{2}\right) \quad \forall 0<t_{1} \leq t_{2}<+\infty
$$

Note that

$$
\begin{gathered}
S_{f}\left(t_{1}\right)= \\
\rho C_{V} \iint_{\mathrm{D}} \ln \left[\int_{0}^{t_{1}} \sum_{m, n=0}^{\infty} B_{m n} e^{-\omega_{m n}^{2}\left(t_{1}-\tau\right)} \cos \frac{m \pi x}{a} \cos \frac{n \pi y}{b} d \tau\right] d \mathrm{~A} \\
S_{f}\left(t_{2}\right)= \\
\rho C_{V} \iint_{\mathrm{D}} \ln \left[\int_{0}^{t_{1}} \sum_{m, n=0}^{\infty} B_{m n} e^{-\omega_{m n}^{2}\left(t_{1}-\tau\right)} \cos \frac{m \pi x}{a} \cos \frac{n \pi y}{b} d \tau\right] d \mathrm{~A}
\end{gathered}
$$

The following inequality is thus obtained:

$$
\iint_{\mathrm{D}} \ln \left[\int_{0}^{t_{2}} \sum_{m, n=0}^{\infty} B_{m n} e^{-\omega_{m n}^{2}\left(t_{2}-\tau\right)} \cos \frac{m \pi x}{a} \cos \frac{n \pi y}{b} d \tau\right] d \mathrm{~A}
$$




$$
\leq \iint_{D} \ln \left[\int_{0}^{t_{2}} \sum_{m, n=0}^{\infty} B_{m n} e^{-\omega_{m n}^{2}\left(t_{2}-\tau\right)} \cos \frac{m \pi x}{a} \cos \frac{n \pi y}{b} d \tau\right] d \mathrm{~A}
$$

Also,

$$
\begin{gathered}
S_{f}(0)=\lim _{t \rightarrow+0} S_{f}(0)=\rho C_{V} \int_{0}^{b} \int_{0}^{a} \lim \ln \left[T_{f}(x, y, t)\right] d x d y \\
=\rho C_{V} \iint_{D} \ln \left[\lim _{t \rightarrow+0} T_{f}(x, y, t)\right] d \mathrm{~A}=\rho C_{V} \iint_{\mathrm{D}} \ln [0] d \mathrm{~A}=-\infty \\
S_{f}(+\infty)=\rho C_{V} \iint_{\mathrm{D}} \ln \left[\lim _{t \rightarrow+\infty} T_{f}(x, y, t)\right] d \mathrm{~A} \\
=\rho C_{V} \iint_{\mathrm{D}} \ln \left[\operatorname { l i m } _ { t \rightarrow + \infty } \left[\int_{0}^{t} \sum_{m, n=0}^{\infty} B_{m n} e^{-\omega_{m n}^{2}(t-\tau)}\right.\right. \\
\left.\left.\cos \frac{m \pi x}{a} \cos \frac{n \pi y}{b} d \tau\right]\right] d \mathrm{~A}
\end{gathered}
$$

As $S_{f}(0) \leq S_{f}(\infty)$, one has,

$$
\begin{gathered}
\rho C_{V} \iint_{\mathrm{D}} \ln \left[\operatorname { l i m } _ { t \rightarrow + \infty } \left[\int_{0}^{t} \sum_{m, n=0}^{\infty} B_{m n} e^{-\omega_{m n}^{2}(t-\tau)}\right.\right. \\
\left.\left.\cos \frac{m \pi x}{a} \cos \frac{n \pi y}{b} d \tau\right]\right] d \mathrm{~A} \geq-\infty
\end{gathered}
$$

Let's now examine $S_{f}(\infty)$ in details. By Eq.(41),

$$
T_{f}(x, y, t)=\int_{0}^{t} U(x, y, t, \tau) d \tau
$$

where $U(x, y, t, \tau)$ is the solution of (40) and reads [Eq. (41)],

$$
U(x, y, t, \tau)=\sum_{m, n=0}^{\infty} B_{m n} e^{-\omega_{m n}^{2}(t-\tau)} \cos \frac{m \pi x}{a} \cos \frac{n \pi y}{b}
$$

As

$$
\lim _{t \rightarrow+\infty} U(x, y, t, \tau)=B_{00}=\frac{1}{A} \iint_{D} f(x, y, \tau) d A
$$

Therefore, for sufficiently large $t_{0}$,

$$
T_{f}\left(x, y, t_{0}\right)=\int_{0}^{t_{0}} U(x, y, t, \tau) d \tau=\int_{0}^{t_{0}} \frac{1}{A} \iint_{D} f(x, y, \tau) d A d \tau
$$

and

$$
\begin{aligned}
S_{f}\left(t_{0}\right) & =\rho C_{v} \iint_{D} \ln \left[\int_{0}^{t_{0}} \frac{1}{A} \iint_{D} f(x, y, \tau) d A d \tau\right] d A \\
& =\rho C_{V} A \ln \left[\int_{0}^{t_{0}} \frac{1}{A} \iint_{D} f(x, y, \tau) d A d \tau\right]
\end{aligned}
$$

Consider now the source with a positive average over the rectangle so that for any time instant $\tau$, there exists a positive value $\varepsilon$ such that

$$
\frac{1}{A} \iint_{D} f(x, y, \tau) d A>\varepsilon>0
$$

or

$$
\frac{1}{\mathrm{~A}} \int_{0}^{t_{0}} d \tau \iint_{D} f(x, y, \tau) d A>\varepsilon t_{0}
$$

$\mathrm{T}_{\text {hus, }}$

$$
S_{f}\left(t_{0}\right)>\rho C_{v} A \ln \left(\varepsilon t_{0}\right)
$$

As $\varepsilon>0, \varepsilon t_{0}$ can be sufficiently large for sufficiently large $t_{0}$. Note also that $\ln x$ always increases with $x$. Therefore, with Eq. (69),

$$
\forall M>0, \exists t_{0} \text {, when } t_{0}<t, M<S_{f}(t)
$$

That is

$$
\lim _{t \rightarrow+\infty} S_{f}(t)=S_{f}(+\infty)=+\infty
$$

2.3 Heat conduction driven by the initial temperature distribution and the internal source

The temperature field subjected to the effect of both initial temperature distribution and the internal source is, by the superimposition principle,

$$
T_{\varphi f}(x, y, t)=T_{\varphi}(x, y, t)+T_{f}(x, y, t)
$$

With Eqs. (15) and (45), we have

$$
\begin{gathered}
T_{\varphi f}(x, y, t)= \\
\sum_{m, n=0}^{\infty} A_{m n} e^{-\omega_{m n}^{2} t} \cos \frac{m \pi x}{a} \cos \frac{n \pi y}{b}+ \\
\int_{0}^{t} \sum_{m, n=0}^{\infty} B_{m n} e^{-\omega_{m n}^{2}(t-\tau)} \cos \frac{m \pi x}{a} \cos \frac{n \pi y}{b} d \tau \\
=\sum C_{m n} e^{-\omega_{m n}^{2} t}+\int_{0}^{t} \sum D_{m n} e^{-\omega_{m n}^{2}(t-\tau)} d \tau
\end{gathered}
$$

where

$$
\begin{aligned}
& \sum C_{m n}=\sum_{m, n=0}^{\infty} A_{m n} \cos \frac{m \pi x}{a} \cos \frac{n \pi y}{b} \\
& \sum D_{m n}=\sum_{m, n=0}^{\infty} B_{m n} \cos \frac{m \pi x}{a} \cos \frac{n \pi y}{b}
\end{aligned}
$$

The total entropy in the rectangle at time instant $t$ is, which is also the entropy generation up to time instant $t, 1,2,6$

$$
S_{\varphi f}(t)=\rho C_{V} \int_{0}^{b} \int_{0}^{a} \ln \left[T_{\varphi f}(x, y, t)\right] d x d y
$$


$=\rho C_{V} \iint_{\mathrm{D}} \ln \left[\sum C_{m n} e^{-\omega_{m n}^{2} t}+\int_{0}^{t} \sum D_{m n} e^{-\omega_{m n}^{2}(t-\tau)} d \tau\right] d \mathrm{~A}(74)$

Also,

$$
\begin{aligned}
& \lim _{t \rightarrow+\infty} S_{\varphi f}(t) \\
& =\rho C_{V} \int_{0}^{b} \int_{0}^{a} \ln \left[A_{00}+\lim _{t \rightarrow+\infty} T_{f}(x, y, t)\right] d x d y \\
& =\rho C_{V} \iint_{\mathrm{D}} \ln \left[\frac{1}{A} \iint_{\mathrm{D}} \varphi(x, y) d \mathrm{~A}+\right. \\
& \left.\lim _{t \rightarrow \infty} \int_{0}^{t} \sum D_{m n} e^{-\omega_{m n}^{2}(t-\tau)} d \tau\right] d \mathrm{~A}
\end{aligned}
$$

Note that

$$
\begin{gathered}
\frac{d S_{\varphi f}(t)}{d t}=\rho C_{V} \int_{0}^{b} \int_{0}^{a} \frac{\partial}{\partial t}\left[\ln T_{\varphi f}(x, y, t)\right] d x d y \\
=\rho C_{V} \iint_{D} \frac{1}{T_{\varphi}(x, y, t)+T_{f}(x, y, t)}\left[\frac{\partial T_{\varphi}(x, y, t)}{\partial t}+\frac{T_{f}(x, y, t)}{\partial t}\right] d \mathrm{~A}
\end{gathered}
$$

By Eqs. (20) and (55), one has

$$
\begin{aligned}
& \frac{\partial T_{\varphi}(x, y, t)}{\partial t}+\frac{\partial T_{f}(x, y, t)}{\partial t} \\
= & \sum C_{m n}\left(-\omega_{m n}^{2}\right) e^{-\omega_{m n}^{2} t}+ \\
& \int_{0}^{t} \sum D_{m n}\left(-\omega_{m n}^{2}\right) e^{-\omega_{m n}^{2}(t-\tau)} d \tau+f(x, y, t)
\end{aligned}
$$

By the second law of thermodynamics that requires $d S_{\varphi f}(t) / d t \geq 0$, one has

$$
\iint_{D}^{\sum C_{m n}\left(-\omega_{m n}^{2}\right) e^{-\omega_{m n}^{2} t}+\int_{0}^{t} \sum D_{m n}\left(-\omega_{m n}^{2}\right) e^{-\omega_{m n}^{2}(t-\tau)} d \tau+f(x, y, t)} d A \geq 0
$$

Note that

$$
\begin{aligned}
& S_{\varphi f}\left(t_{1}\right)=\rho C_{V} \int_{0}^{b} \int_{0}^{a} \ln \left[T_{\varphi f}\left(x, y, t_{1}\right)\right] d x d y=\rho C_{V} \iint_{\mathrm{D}} \ln \left[T_{\varphi f}\left(x, y, t_{1}\right)\right] d \mathrm{~A} \\
& S_{\varphi f}\left(t_{2}\right)=\rho C_{V} \int_{0}^{b} \int_{0}^{a} \ln \left[T_{\varphi f}\left(x, y, t_{2}\right)\right] d x d y=\rho C_{V} \iint_{\mathrm{D}} \ln \left[T_{\varphi f}\left(x, y, t_{2}\right)\right] d \mathrm{~A}
\end{aligned}
$$

By applying

$$
S_{\varphi f}\left(t_{1}\right) \leq S_{\varphi f}\left(t_{2}\right), \quad \forall 0<t_{1} \leq t_{2}<+\infty
$$

one obtains

$$
\iint_{\mathrm{D}} \ln \left[\sum C_{m n} e^{-\omega_{m n}^{2} t_{1}}+\int_{0}^{t} \sum D_{m n} e^{-\omega_{m n}^{2}\left(t_{1}-\tau\right)} d \tau\right] d \mathrm{~A} \leq \iint_{\mathrm{D}} \ln \left[\left[\sum C_{m n} e^{-\omega_{m n}^{2} t_{2}}+\int_{0}^{t} \sum D_{m n} e^{-\omega_{m n}^{2}\left(t_{2}-\tau\right)} d \tau\right]\right] d \mathrm{~A}
$$




$$
\begin{aligned}
S_{\varphi f}(t) & =\rho C_{V} \int_{0}^{b} \int_{0}^{a} \ln \left[T_{\varphi}(x, y, t)+T_{f}(x, y, t)\right] d x d y \\
& =\rho C_{V} \iint_{D} \ln \left\{\frac{1}{A} \iint_{D} \varphi(x, y) d \mathrm{~A}\right. \\
& \left.+\left[\lim _{t \rightarrow \infty}\left[\int_{0}^{t} \frac{1}{A} \iint f(x, y, t) d A d \tau\right]\right]\right\} d \mathrm{~A} \\
& \geq \rho C_{V} \iint_{\mathrm{D}} \ln \left[\frac{1}{A} \iint_{\mathrm{D}} \varphi(x, y) d \mathrm{~A}+\varepsilon t\right] d \mathrm{~A}
\end{aligned}
$$

As $\varepsilon>0$, $\varepsilon t$ can be sufficiently large for sufficiently large $t$. Note also that $\ln x$ always increases with $x$. Eq. (86) thus leads to

$$
\forall M>0, \exists t_{0} \text {, when } t_{0}<t, M<S_{\varphi f}(t)
$$

that is

$$
\lim _{t \rightarrow+\infty} S_{\varphi f}(t)=S_{f}(+\infty)=+\infty
$$

\section{Discussion}

With an adiabatic boundary, as time tends to infinity, the temperature would become uniform for the heat conduction driven only by the initial temperature distribution, but not when there is any positivelyaveraged internal source. As time tends to infinity, the entropy generation from the heat conduction in adiabatic rectangles has a bounded limit [Eq. (17)] when the heat conduction is initiated by the initial temperature distribution. This is not valid anymore [Eqs. (70) and (87)] when the heat conduction includes an internal source with a positive average over the rectangle. This result is also valid in other systems. ${ }^{37}$ As the entropy generation degrades the energy quality, ${ }^{7}$ the unbounded entropy generation will cause serious problems. We must thus constrain, as far as possible, the use and of technologies that depend on positive average heat generation, such as electric-resistance heating, nuclear fission and exothermic chemical reactions.

Table 1 lists the nine integral inequalities from applying the second law of thermodynamics to the heat conduction in rectangles. Despite of the similar form, they differ from their 1D counterparts in literature $^{6}$ due to different temperature fields. One of these inequalities [Eq. (28)] has also been proven mathematically. For their fundamental nature (from the three fundamental laws: the second law of thermodynamics, the first law of thermodynamics and the Fourier law of heat conduction via the heat-conduction equation), they are important both for studying heat-conduction equations and for assessing accuracy of solutions from analytical, numerical and experimental approaches. Application of Eq. (28) to the total multiplicity $\Omega$ of a macrostate, the number of microstates that compose the macrostate in the Boltzmann entropy, has also led to Eqs. (38) and (39) showing that the entropy average over any domain can never be larger than the entropy calculated with the average multiplicity over the domain. Table 2 lists

\begin{tabular}{|c|c|c|}
\hline $\begin{array}{c}\text { Heat } \\
\text { conduction }\end{array}$ & $\begin{array}{l}\text { Second law of } \\
\text { thermodynamics }\end{array}$ & Inequalities \\
\hline \multirow{3}{*}{$\begin{array}{l}\text { By initial } \\
\text { temperature } \\
\text { distribution }\end{array}$} & $\frac{d S_{\varphi}(t)}{d t} \geq 0$ & $\iint_{D} \frac{\sum_{m, n=0}^{\infty} A_{m n}\left(-\omega_{m n}^{2}\right) e^{-\omega_{m n}^{2} t} \cos \frac{m \pi x}{a} \cos \frac{n \pi y}{b}}{\sum_{m, n=0}^{\infty} A_{m n} e^{-\omega_{m n}^{2} t} \cos \frac{m \pi x}{a} \cos \frac{n \pi y}{b}} d \mathrm{~A} \geq 0$ \\
\hline & $S_{\varphi}\left(t_{1}\right) \leq S_{\varphi}\left(t_{2}\right)$ & $\begin{aligned} & \iint_{D} \ln \left(\sum_{m, n=0}^{\infty} A_{m n} e^{-\omega_{m n}^{2} t_{1}} \operatorname{coh} \frac{m \pi x}{a} \operatorname{coh} \frac{n \pi y}{b}\right) d A \\
\leq & \iint_{D} \ln \left(\sum_{m, n=0}^{\infty} A_{m n} e^{-\omega_{m n}^{2} t_{2}} \operatorname{coh} \frac{m \pi x}{a} \operatorname{coh} \frac{n \pi y}{b}\right) d A\end{aligned}$ \\
\hline & $\begin{array}{l}S_{\varphi}(0) \\
\leq S_{\varphi}(+\infty)\end{array}$ & $\frac{1}{A} \iint_{\mathrm{D}} \ln \varphi(x, y) d \mathrm{~A} \leq \ln \left[\frac{1}{A} \iint_{\mathrm{D}} \varphi(x, y) d \mathrm{~A}\right]$ \\
\hline \multirow[b]{2}{*}{$\begin{array}{l}\text { By internal } \\
\text { source }\end{array}$} & $\frac{d S_{f}(t)}{d t} \geq 0$ & $\iint_{D} \frac{\int_{0}^{t} \sum D_{m n}\left(-\omega_{m n}^{2}\right) e^{-\omega_{m n}^{2}(t-\tau)} d \tau+f(x, y, t)}{\int_{0}^{t} \sum_{m, n=0}^{\infty} B_{m n} e^{-\omega_{m n}^{2}(t-\tau)} \cos \frac{m \pi x}{a} \cos \frac{n \pi y}{b} d \tau} d A \geq 0$ \\
\hline & $S_{f}\left(t_{1}\right) \leq S_{f}\left(t_{2}\right)$ & $\begin{aligned} & \iint_{D} \ln \left[\int_{0}^{t_{1}} \sum_{m, n=0}^{\infty} B_{m n} e^{-\omega_{m n}^{2}\left(t_{1}-\tau\right)} \operatorname{coh} \frac{m \pi x}{a} \operatorname{coh} \frac{n \pi y}{b} d \tau\right] d A \\
\leq & \iint_{D} \ln \left[\int_{0}^{t_{2}} \sum_{m, n=0}^{\infty} B_{m n} e^{-\omega_{m n}^{2}\left(t_{2}-\tau\right)} \operatorname{coh} \frac{m \pi x}{a} \operatorname{coh} \frac{n \pi y}{b} d \tau\right] d A\end{aligned}$ \\
\hline
\end{tabular}
and $\frac{1}{A} \iint_{\mathrm{D}} \ln \varphi(x, y) d \mathrm{~A}$ with $\mathrm{a}=1 \mathrm{~m}, \mathrm{~b}=2 \mathrm{~m}$ for four typical $\ln \left[\frac{1}{A} \iint_{\mathrm{D}} \varphi(x, y) d \mathrm{~A}\right]$ $\varphi(x, y)$, and further demonstrates the correctness of Eq. (28). Equations (28), (38) and (39) are classical and have been acquired by the other approach in the literature. The present work develops a new approach,

Table 1 Integral inequalities developed in present work. 
Table 1

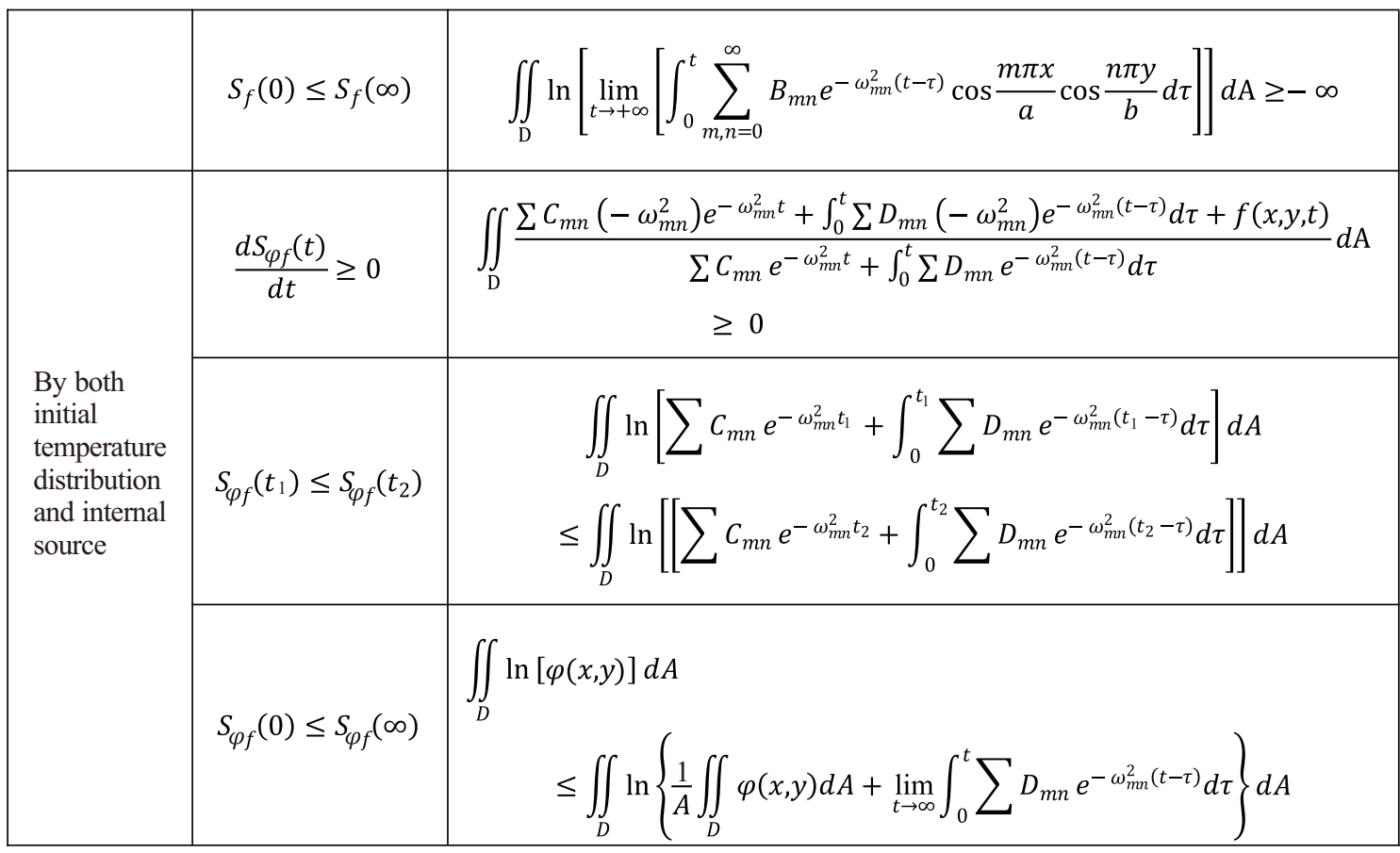

Table 2 Comparison between $\frac{1}{A} \iint_{\mathrm{D}} \ln \varphi(x, y) d \mathrm{~A}$ and $\ln \left[\frac{1}{A} \iint_{\mathrm{D}} \varphi(x, y) d \mathrm{~A}\right](a=2 \mathrm{~m}, b=1 \mathrm{~m})$.

\begin{tabular}{|c|c|c|c|c|}
\hline$\varphi(x, y)$ & $\ln \varphi(x, y)$ & $\frac{1}{A} \iint_{D} \ln \varphi(x, y) d A$ & $\ln \left[\frac{1}{A} \iint_{D} \varphi(x, y) d \mathrm{~A}\right]$ & $\begin{array}{l}\frac{1}{A} \iint_{\mathrm{D}} \ln \varphi(x, y) d \mathrm{~A} \\
\leq \ln \left[\frac{1}{A} \iint_{\mathrm{D}} \varphi(x, y) d \mathrm{~A}\right.\end{array}$ \\
\hline $\begin{array}{c}c \text { (any } \\
\text { positive } \\
\text { constant) }\end{array}$ & $\ln c$ & $\ln c$ & $\ln c$ & YES \\
\hline$e^{x}$ & $x$ & 1 & $\ln \left[\frac{1}{2}\left(e^{2}-1\right)\right]=1.16$ & YES \\
\hline$e^{y}$ & $y$ & 0.5 & $\ln (e-1)=0.54$ & YES \\
\hline$e^{x-y}$ & $x-y$ & 0.5 & $\begin{array}{r}\ln \left[\frac{1}{2}\left(e^{2}+e^{-1}-e-1\right)\right] \\
=0.70\end{array}$ & YES \\
\hline
\end{tabular}


the second-law approach, to obtain them, thus building their relation to the second law of thermodynamics. The second-law approach works also for developing new mathematical inequalities.

\section{Concluding remarks}

As the time tends to infinity,the entropy generation during the heat conduction in adiabatic rectangles evolves into a finite value when the heat conduction comes from the initial temperature distribution, but infinity when the conduction contains any heat source of positive average over the rectangle. Therefore, it is critical to use as less technologies as possible that could yield positive average heat generation and to develop their replacing technologies, for preventing unbounded entropy generation and thus exergy or energy/quality destruction.

The nine inequalities are obtained by applying the second law of thermodynamics to the heat conduction in rectangles: eight being new and the other one being classical. They are capable for examining correctness and accuracy of various studies of heat conduction processes. An application of one of these nine inequalities to the total multiplicity of a macrostate in the Boltzmann entropy concludes that the entropy average over any domain can never be larger than the entropy calculated with the average multiplicity over the domain.

\section{Acknowledgements}

The financial support from the Research Grants Council of Hong Kong (GRF 17204718, 17237316 and 17211115) isgratefully acknowledged. The work is also supported in part by the Zhejiang Provincial, Hangzhou Municipal and Lin'an County Governments.

\section{Author Contributions}

$\mathrm{X} . \mathrm{T}$ and $\mathrm{L}$. W. conceived the project. X. T. and L. W. designed the project. X. T. performed mathematical derivation. X. T. and L. W. analyzed the results. X. T. and L. W. wrote the manuscript. L. W. supervised the study.

\section{Additional Information}

Competing financial interests: The authors declare no financial and non-financial competing interests.

\section{References}

1. Y. A. Cengel and M. A. Boles, 2011, Thermodynamics: An Engineering Approach, $7^{\text {th }}$ ed, McGraw-Hill, New York.

2. A. Bejan, 2016, Advanced Engineering Thermodynamics, $4^{\text {th }}$ ed, Wiley, Newark.

3. L. Q. Wang, X. S. Zhou and X. H. Wei, Heat Conduction:Mathematical Models and Analytical Solutions, Springer-Verlag, Heidelberg, 2008.

4. A. Bejan, Heat Transfer, John Wiley \& Sons, New York, 1993.

5. L. Q. Wang, Int. J. Heat Mass Tran., 1994, 37, 2627-2634.

6. X. W, Tian, X. Lai, P. A. Zhu and L. Q. Wang, P. Roy. Soc. A, 2016, 472, 20160362.
7. L. Q. Wang, Int. J. Heat Mass Tran., 1998, 41, 1869-1871.

8. X. W. Tian and L. Q. Wang, Int. J. Heat Mass Tran., 2018, 121, 1137-1145.

9. M. Lange, M. Roeb, C. Sattler and R. Pitz-Paal, Entropy, 2016, 18(1), 24.

10. X. J. Zhu, X. Du, Y. Q. Ding and Q. G. Qiu, Int. J. Heat Mass Tran., 2017, 114, 20-30.

11. Y. Ji, H. C. Zhang, X. Yang and L. Shi, Entropy, 2017, 19 (3), 108.

12. E. Guelpa and V. Verda, Entropy, 2017, 19 (8), 433.

13. Z. X. Li and Z. Y. Guo, Advances in Transport Phenomena 2010 (Ed. By L. Q. Wang), 1-91, Springer-Verlag, Heidelberg, 2011.

14. M. T. Xu, J. F. Guo and X. F. Li, Advances in Transport Phenomena2011 (Ed. By L. Q. Wang), 63-167, Springer-Verlag, Heidelberg, 2014.

15. D. L. Goodstein, Thermal Physics: Energy and Entropy, Cambridge University Press, Cambridge, 2015.

16. G. F. Naterer and J. A. Camberos, Entropy-based Design and Analysis of Fluids Engineering Systems, CRC Press, Boca Raton, 2008.

17. A. Bejan, Entropy Generation Minimization: the Method of Thermodynamic Optimization of Finite-Size Systems and Finite-time Processes, CRC Press, New York, 1996.

18. M. M. Rashid and M. A. Abbas, Entropy, 2017, 19 (8), 414.

19. H. Wang, D. Lin, X. R. Su and X. Yuan, Entropy, 2017, 19 (7), 324.

20. T. Choudhary, S. Sanjay, Energy, 2017, 134, 1013-1028.

21. T. Hayat, S. Nawaz, A. Alsaedi and M. Rafiq, Entropy, 2016,18 (10), 355

22. I. Dinçer and M. A.Rosen, Exergy Analysis of Heating, Refrigerating and Air Conditioning: Methods and Applications, Elsevier, Amsterdam, 2015.

23. V. M. Brodyanbsky, M. V. Sorin and P. Le Goff, The Efficiency of Industrial Processes : Exergy Analysis and Optimization, Elsevier, Amsterdam, 1994.

24. D. H. Yang, Exergy and Energy-order Analyses, Science Press, Beijing, 1986.

25. N. Freidoonimehr, M. M. Rashidi, S. Abelman and G. Lorenzini, Entropy, 2016, 18(5), 131.

26. C. E. Damian-Ascencio, A. Saldana-Robles, A. Hernandez-Guerrero and S. Cano-Andrade, Energy, 2017, 133, 306-316.

27. M. A. Sheremet, H. F. Oztop, I. Pop and N. Abu-Hamdeh, Entropy, 2016, 18 (1), 9 .

28. V. P. Singh, Entropy Theory in Hydrologic Science and Engineering, McGraw-Hill, New York, 2015.

29. Q. Jia, M. B. Muhammad, A. A. Munawwar, M. R. Mohammad and E. A. Mohamed, Entropy, 2016, 18, 123.

30. H. A. Muhammad, F. Navid, N. Foad and M. R. Mohammad, Adv. Pow Technol., 2017, 26, 542-552.

31. Y. A. Cengel, Y. A. Cengel, Heat and Mass Transfer: An Engineering Approach, 3rd ed, McGraw-Hill, New York, 2007.

32. E. F. Beckenbach and R. Bellman, Inequalities, Springer-Verlag, Berlin, 1965.

33. G. H. Hardy, J. E. Littlewood and G. P'olya, Inequalities, Cambridge University Press, Cambridge, 1934.

34. D. S. Lemons, A Student's Guide to Entropy, Cambridge University Press, Cambridge, 2013.

35. I. Ford, Statistical Physics: An Entropic Approach, Wiley, Hoboken, 2013.

36. T. Downarowicz, 2011, Entropy in Dynamical Systems, Cambridge University Press, New York.

37. X. W. Tian, 2018, PhD thesis, Entropy Generation in Heat Conduction and Engineering Embolic Micro-particles with Electrospray, the University of Hong Kong.

Publisher's Note Engineered Science Publisher remains neutral with regard to jurisdictional claims in published maps and institutional affiliations. 